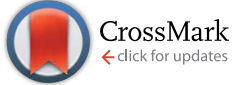

Cite this: RSC Adv., 2017, 7, 17346

Received 25th February 2017

Accepted 13th March 2017

DOI: $10.1039 / c 7 r a 02346 d$

rsc.li/rsc-advances

\section{Integration of heterologous 4-hydroxybenzoic acid transport proteins in Rhodobacter sphaeroides for enhancement of coenzyme $Q_{10}$ production}

\author{
Feng Qi, $\uparrow^{\text {*a }}$ Limei Zou, $\uparrow^{\mathrm{a}}$ Xianzhang Jiang, ${ }^{a}$ Shaoli Cai, ${ }^{\mathrm{b}}$ Mingliang Zhang, ${ }^{\mathrm{a}}$ \\ Xuebing Zhao ${ }^{\mathrm{C}}$ and Jianzhong Huang*a
}

\begin{abstract}
The highly redox-active compound, Coenzyme $Q_{10}\left(C_{0} Q_{10}\right)$, has attracted increasing interest due to its clinical benefits and important applications in the pharmaceutical, food and health industries. Although the metabolic pathways involved in $\mathrm{CoQ}_{10}$ biosynthesis are known, very few reports are available concerning the development of engineering strategies to enhance $\mathrm{CoQ}_{10}$ production in the natural producer Rhodobacter sphaeroides. In this work, three membrane transport proteins, AcPcaK, KpPcaK, and CgPcaK from different organisms (Acinetobacter calcoaceticus, Klebsiella pneumoniae and Corynebacterium glutamicum, respectively) were individually heterologously expressed in $R$. sphaeroides GY-2 to enhance the uptake of extracellular 4-hydroxybenzoic acid (4HBA), an important intermediate of $\mathrm{CoQ}_{10}$ synthesis. Each of the PcaK open reading frames (ORFs) was inserted behind an RFP gene to weaken their expression in order to avoid inhibition of cell growth. The transport efficiency and consumption of $4 \mathrm{HBA}$, as well as $\mathrm{CoQ}_{10}$ productivity of the resulting recombinant $R$. sphaeroides $\mathrm{GY}-2$ strains has been studied. The $\mathrm{CoQ}_{10}$ productivity of RS-CgPcaK and RS-KpPcaK was effectively improved by the addition of external 4HBA, reaching maximum values of 17.45 and $18.06 \mathrm{mg} \mathrm{g}^{-1} \mathrm{DCW}(18.05 \%$ and $20.82 \%$ higher than $R$. sphaeroides GY-2), respectively. The strategy used in this study proved effective for enhancing the biotechnological production of $\mathrm{CoQ}_{10}$ and thus holds promise for further improvements, including industrial applications.
\end{abstract}

\section{Introduction}

The ubiquinone Coenzyme $\mathrm{Q}_{10}\left(\mathrm{CoQ}_{10}\right)$ is composed of a benzoquinone "head" group and a side chain composed of ten isoprenoid units, which distinguishes it from other $\operatorname{CoQ}_{n}$ cognates. $\mathrm{CoQ}_{10}$ is embedded within the hydrophobic domain of the phospholipid bilayer of cytoplasmic and organellar membranes, where it functions as an electron carrier in the respiratory chain. ${ }^{\mathbf{1 , 2}} \mathrm{CoQ}_{10}$ plays an important role as a redoxactive molecule in the formation of disulfide bonds, as an antioxidant for removing harmful reactive oxygen species, and as a controller of the cellular redox status. ${ }^{3-5}$ The clinical benefits of $\mathrm{CoQ}_{10}$ supplementation include the prevention of heart failure and acute myocardial infarction in cardiovascular diseases, as well as positive effects in mitochondrial and certain

${ }^{a}$ Engineering Research Center of Industrial Microbiology of Ministry of Education, College of Life Sciences, Fujian Normal University, Fuzhou 350117, Fujian, China. E-mail: f.qi@fjnu.edu.cn; hjz@fjnu.edu.cn; Fax: +86 591 22868212; Tel: +86 591 22868212

${ }^{b}$ Biomedical Research Center of South China, Fujian Normal University, Fuzhou 350117, Fujian, China

'Institute of Applied Chemistry, Department of Chemical Engineering, Tsinghua University, Beijing 100084, China

$\dagger$ Feng Qi and Limei Zou contributed equally to this work. neurodegenerative diseases and diabetes. ${ }^{6,7}$ Furthermore, $\mathrm{CoQ}_{10}$ acts as a special regulator of the genes involved in cholesterol metabolism and inflammatory responses. ${ }^{8}$ Over the past two decades, there has been growing interest in the research and production of $\mathrm{CoQ}_{10}$ due to these healthpromoting effects which it exerts either as a food additive or medicine. Some prokaryotic microorganisms, such as Rhodobacter sphaeroides and Agrobacterium tumefaciens, have been studied by researchers as natural producers of $\mathrm{CoQ}_{10} \cdot{ }^{9-11} \mathrm{In}$ prokaryotic cells, the biosynthesis of $\mathrm{CoQ}_{10}$ utilizes three combined pathways. The benzoquinone "head" is synthesized from 4-hydroxybenzoic acid (4HBA) in the shikimate pathway, whereas the isoprenoid side chain is synthesized by an IPP synthesis enzyme, using ten isopentenyl pyrophosphate (IPP) units derived from pyruvate and glyceraldehyde-3-phosphate via the methylerythritol phosphate (MEP) pathway. Finally, $\mathrm{CoQ}_{10}$ is assembled by linking the isoprenoid side chain to the benzoquinone head in the ubiquinone pathway.

This detailed knowledge of the key genes and metabolic pathways involved in $\mathrm{CoQ}_{10}$ biosynthesis has made it possible to develop rational genetic engineering strategies to enhance the $\mathrm{CoQ}_{10}$ productivity of prokaryotic microorganisms. For instance, improved $\mathrm{CoQ}_{10}$ production was obtained by overexpressing the native rate-limiting enzymes of the MEP and 
ubiquinone pathways in $R$. sphaeroides. ${ }^{11,12}$ However, most relevant studies were carried out using $E$. coli as the $\operatorname{CoQ}_{10}$ producer, owing to its ease of genetic manipulation., ${ }^{2,5,13-15}$ The specific productivity of $\mathrm{CoQ}_{10}$ achieved by these metabolically engineered $E$. coli strains was very low and not comparable to the natural $\mathrm{CoQ}_{10}$ producers such as $R$. sphaeroides, $A$. tumefaciens and $R$. radiobacter. ${ }^{\mathbf{1 6 , 1 7}}$ Thus, it seems particularly attractive and practical to directly genetically modify $R$. sphaeroides and improve upon its intrinsically high $\mathrm{CoQ}_{10}$ titer.

In the present study, we focused on increasing the supplementation and availability of 4HBA, the initial precursor of the ubiquinone pathway, which we predicted would result in enhanced $\mathrm{CoQ}_{10}$ production in $R$. sphaeroides. PcaK is a transporter and chemoreceptor membrane protein which is known to facilitate the transmembrane transport of $4 \mathrm{HBA}$ in Pseudomonas putida and Klebsiella pneumoniae.$^{\mathbf{1 8 , 1 9}}$ In this study, three PcaK homologs, those derived from Acinetobacter calcoaceticus ATCC 14987 (AcPcaK, Genbank accession no. KUM11311.1), Klebsiella pneumoniae ATCC 33495 (KpPcaK, Genbank accession no. KUP78028.1) and Corynebacterium glutamicum ATCC 13032 (CgPcaK, Genbank accession no. BAV23994.1), have been expressed in $R$. sphaeroides GY-2, a high-yield CoQ10 producing mutant strain obtained from $R$. sphaeroides 2.4.1 (ATCC 17023) in our previous work. We sought to improve the $\mathrm{CoQ}_{10}$ productivity in $R$. sphaeroides GY-2 by extracellularly supplementing 4HBA, which would then be transported into the cells by the overexpressed PcaK. However, the growth and metabolism of many microorganisms can be greatly inhibited by species-specific concentrations of $4 \mathrm{HBA}^{20}$ To circumvent this obstacle, each of the PcaK reading frames has been inserted behind a red fluorescent protein (RFP) gene under the control of the tac promoter (Fig. 1), in order to control for the effects of
4HBA on biomass accumulation and the utilization of supplemented 4HBA for the synthesis of $\mathrm{CoQ}_{10}$ in the resulting recombinant $R$. sphaeroides strains. This work thus provides a novel genetic engineering strategy for the improvement of $\mathrm{CoQ}_{10}$ production in $R$. sphaeroides and other industrially relevant microorganisms.

\section{Experimental}

\section{Strains, media and culture conditions}

The strains and plasmids used in this study are listed in Table 1. E. coli strains were cultivated at $37{ }^{\circ} \mathrm{C}$ in Luria-Bertani (LB) medium supplemented with $50 \mathrm{mg} \mathrm{ml}^{-1}$ of kanamycin sulfate when necessary. E. coli $\mathrm{DH} 5 \alpha$ was used for cloning and plasmid construction and $E$. coli S17-1 was used for bi-parental conjugation. $R$. sphaeroides GY-2 is a mutant strain derived from $R$. sphaeroides 2.4.1 (ATCC 17023) via screening, which has a high yield of $\mathrm{CoQ}_{10}$. The specific culture medium used for $R$. sphaeroides GY-2 comprised $4 \mathrm{~g} \mathrm{l}^{-1}$ glucose, $8 \mathrm{~g} \mathrm{l}^{-1}$ yeast extract, $5 \mathrm{~g} \mathrm{l}^{-1}$ $\mathrm{NaCl}, 2 \mathrm{~g} \mathrm{l}^{-1} \mathrm{MgCl}_{2}, 3 \mathrm{~g} \mathrm{l}^{-1} \mathrm{KH}_{2} \mathrm{PO}_{4}, 1 \mathrm{~g} \mathrm{l}{ }^{-1} \mathrm{CaCl}_{2}, 1 \mathrm{~g} \mathrm{l}{ }^{-1}$ glutamic acid, $0.4 \mathrm{~g} \mathrm{l}^{-1}$ aspartic acid, $2 \mathrm{~g} \mathrm{l}^{-1}$ nitrilotriacetic acid, $1 \mathrm{~g}$ $\mathrm{l}^{-1}$ nicotinic acid (Amresco Ltd., Solon, US), and $1 \mathrm{~g} \mathrm{l}^{-1}$ biotin (Sigma-Aldrich, St Louis, US), with an initial pH of 4.5-5.5.

The fermentation medium comprised $32 \mathrm{~g} \mathrm{l}^{-1}$ glucose, $4 \mathrm{~g} \mathrm{l}^{-1}$ corn steep liquor (Beijing Chemical Works, China), $6.5 \mathrm{~g} \mathrm{l}^{-1}$ glutamic acid, $4 \mathrm{~g} \mathrm{l}^{-1}\left(\mathrm{NH}_{4}\right)_{2} \mathrm{SO}_{4}, 3.25 \mathrm{~g} \mathrm{l}^{-1} \mathrm{NaCl}, 10 \mathrm{~g} \mathrm{l}^{-1} \mathrm{MgSO}_{4}$, $1.4 \mathrm{~g} \mathrm{l}^{-1} \mathrm{FeSO}_{4}, 1.2 \mathrm{~g} \mathrm{l}^{-1} \mathrm{KH}_{2} \mathrm{PO}_{4}, 0.06 \mathrm{~g} \mathrm{l}^{-1} \mathrm{MnSO}_{4}, 0.06 \mathrm{~g} \mathrm{l}^{-1}$ $\mathrm{CoCl}_{2}, 8 \mathrm{~g} \mathrm{l}^{-1} \mathrm{CaCO}_{3}, 1 \mathrm{~g} \mathrm{l}^{-1}$ nicotinic acid, and $0.3 \mathrm{~g} \mathrm{l}^{-1}$ biotin.

\section{Construction of vectors}

pBBR1MCS2 is a low-copy-number broad-host-range vector for DNA cloning in Gram-negative bacteria. ${ }^{23}$ The expression

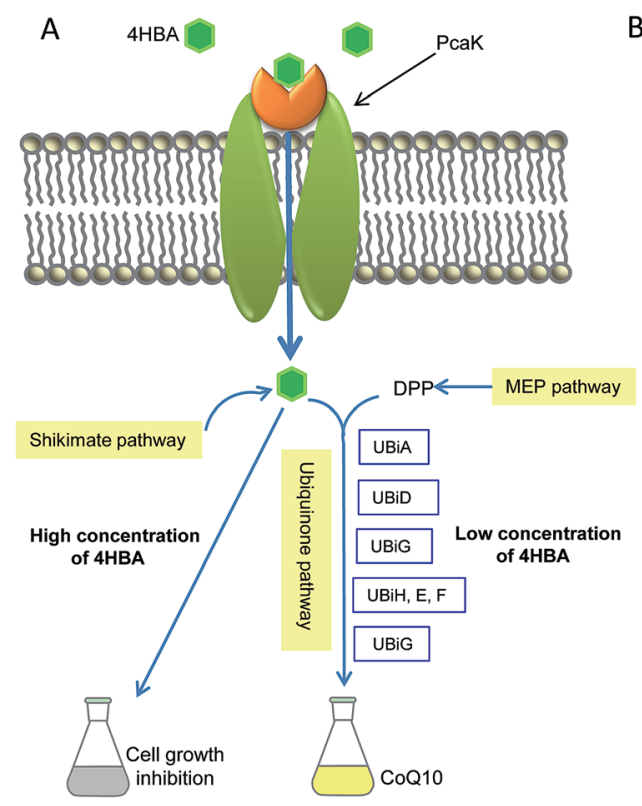

B
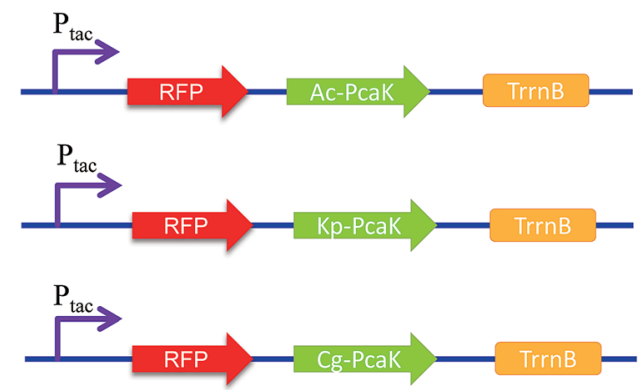

Fig. 1 Schematic presentation of the PcaK functions in $\mathrm{CoQ}_{10}$ biosynthesis. (A) PcaK-mediated membrane transport of 4HBA. (B) Construction of the expressing fragments of PcaK in this study. DPP: decaprenyl diphosphate; 4HBA: 4-hydroxybenzoic acid; UbiA: 4HBA-polyprenyltransferase; UbiD, B, E, F, G, H: quinoid ring modification enzymes. 
Table 1 Strains, plasmids and primers used in this study

\begin{tabular}{|c|c|c|}
\hline Strain & Genotype/description & Reference \\
\hline E. coli $\mathrm{DH} 5 \mathrm{a}$ & $\begin{array}{l}\text { F- } \lambda \text {-endA1 glnV44 thi-1 recA1 relA1 gyrA96 deoR nupG } \Phi 80 \text { dlacZ } \Delta \text { M15 } \\
\Delta \text { (lacZYA-argF)U169, hsdR17(rK- mK+) }\end{array}$ & Invitrogen \\
\hline R. sphaeroides-GY2 & CoQ10 high-yield mutant strain obtained from $R$. sphaeroides 2.4.1 & This study \\
\hline Acinetobacter calcoaceticus & Genomic source of Ac-Pcak & ATCC 14987 \\
\hline Klebsiella pneumoniae & Genomic source of Kp-Pcak & ATCC 33495 \\
\hline Plasmid & Description & Reference \\
\hline pYC6a-RFP & pYC6a derivative containing RFP, $\mathrm{Amp}^{\mathrm{R}}$ & This study \\
\hline pBBR1MCS2 & Low-copy cloning vector, $\operatorname{Kan}^{\mathrm{R}}$ & 22 \\
\hline pBBR1MCS2-tac-RFP & pBBR1MCS2 derivative expression plasmid with the tac promoter and RFP & This study \\
\hline $\begin{array}{l}\text { pBBR1MCS2-tac-RFP derivative } \\
\text { containing Pcak from C. glutamicum }\end{array}$ & pBBR1MCS2-RFP-CgPcak & This study \\
\hline
\end{tabular}

Primers used in this study

\begin{tabular}{ll}
\hline Designation & Sequence $\left(5^{\prime}-3^{\prime}\right)$ \\
\hline acPcaK-F & CCGGAATTCATGGCGAAAACGATAGATGCAA \\
acPcaK-R & CGCGGATCCTTATAGACTTTCTTCGAGCTTTAA \\
kpPcaK-F & CCGGAATTCATGACACAGACTCAACGCCTGGATGTCA \\
kpPcaK-R & CGCGGATCCCTATGACGCTCCGCGCACAGACGCGG \\
cgPcaK-F & CCGGAATTCATGGCGTCAACGACCACCCCAACCCGCG \\
cgPcaK-R & CGCGGATCC TTAAGCGTTCTCGCGATGCCTCAGAGTCTT \\
tRFP-F & ATTCCCCGCGGCACAGCTAACACCACGTCGTC \\
tRFP-R & GAGCCCAAGCTTGAAAGGCCCAGTCTTTCGAC
\end{tabular}

plasmid pBBR1MCS2-tac-RFP is derived from pBBR1MCS2 and pYC6a-RFP. The fragment containing the tac promoter, RFP and the TrrnB terminator was amplified from pYC6a-RFP using primers tRFP-F and tRFP-R, the resulting fragment digested using the restriction endonucleases SacII and HindIII, and ligated into pBBR1MCS2, yielding the plasmid pBBR1MCS2-tacRFP. The genes encoding AcPcak, CgPcak and KpPcak were amplified using the primers listed in Table 1, the resulting fragments digested using the restriction endonucleases EcoRI and BamHI, and ligated into pBBR1MCS2-tac-RFP, yielding pBBR1MCS2-RFP-CgPcak, pBBR1MCS2-RFP-AcPcak, and pBBR1MCS2-RFP-KpPcak, respectively. To avoid the formation of a fusion protein comprising Pcak and RFP, a stop codon was added to the end of the open reading frame of RFP. The Q5 polymerase, endonucleases, and T4 ligase used in this study were all purchased from New England Biolabs (Beijing, China).

\section{Bi-parental conjugation}

The plasmids pBBR1MCS2-tac-RFP, pBBR1MCS2-RFP-CgPcak, pBBR1MCS2-RFP-AcPcak and pBBR1MCS2-RFP-KpPcak were used to transform E. coli S17-1 via electroporation, and the resulting strains were used as plasmid donors. The process of conjugation mating was carried out as described in a previous study. ${ }^{24}$ Briefly, cultures of E. coli S17-1 (donor) and $R$. sphaeroides GY-2 (recipient) were grown to mid-log phase, harvested by centrifugation $(8000 \mathrm{~g}, 5 \mathrm{~min})$ and resuspended in fresh $\mathrm{LB}$ medium. The E. coli S17-1 and $R$. sphaeroides GY-2 cell suspensions were mixed at $1: 6$ and $1: 10$ ratios, the resulting mixtures of the two strains harvested, transferred to an LB agar plate, and incubated for $20-24 \mathrm{~h}$ at $30{ }^{\circ} \mathrm{C}$. The resulting bacterial lawns were harvested by washing with $0.1 \mathrm{ml}$ pre-cooled improved Sistrom's minimal medium ${ }^{25}$ and spread on LB agar plates containing $200 \mathrm{mg} \mathrm{l}^{-1} \mathrm{~K}_{2} \mathrm{TeO}_{3}$ and $50 \mathrm{mg} \mathrm{ml}^{-1}$ kanamycin. Kanamycin-resistant black colonies were isolated, verified, and utilized for further experiments. The recombinant $R$. sphaeroides GY-2 harboring pBBR1MCS2-tac-RFP was used as the control.

\section{Western blot analysis}

Western blot analysis was employed to detect the expression of the recombinant PcaK in $R$. sphaeroides GY-2. Briefly, $R$. sphaeroides cells cultured for $24 \mathrm{~h}$ in the specific medium containing $50 \mathrm{mg} \mathrm{l}^{-1}$ kanamycin were collected, re-suspended, and washed with sterile water. Membrane proteins of the recombinant strains were extracted using the Membrane Extraction Kit (Sigma-Aldrich, St Louis, US) according to the manufacturer's protocol. The resulting crude membrane extracts were further separated by SDS-PAGE on $12 \%$ polyacrylamide gels and transferred to an Immun-Blot assay kit membrane (Bio-Rad, Beijing, China) via semi-dry electrotransfer. The six histidine- 
tagged fusions of PcaK were probed with a mouse anti-His ${ }_{6}$ antibody and a secondary HRP-labeled goat anti-mouse IgG (1 $\mu \mathrm{g} \mathrm{ml}{ }^{-1}$; Amersham, Beijing, China). The PVDF membrane was washed with $1 \times$ TBST $(0.1 \%$ Tween 20$) 4$ times and the blotting result was visualized using a near-infrared fluorescence detector (Bio-Rad, Beijing, China).

\section{$\mathrm{CoQ}_{10}$ production in shake-flask cultures}

The recombinant $R$. sphaeroides GY-2 strains were inoculated and grown in the specific medium at pH 6.4 and $32{ }^{\circ} \mathrm{C}$, under constant orbital shaking at $200 \mathrm{rpm}$ for $24 \mathrm{~h}$. The strains were then transferred to $500 \mathrm{ml}$ baffled flasks containing $150 \mathrm{ml}$ of fermentation medium at $32{ }^{\circ} \mathrm{C}$ and $\mathrm{pH}$ 6.3-6.4 under constant orbital shaking $220 \mathrm{rpm}$ for $48 \mathrm{~h}$. After the fermentation process, 0.5-1 $\mathrm{ml}$ aliquots of the fermentation broth were harvested, combined $200 \mu \mathrm{l} \mathrm{HCl}$ solution $\left(6 \mathrm{~mol} \mathrm{l}^{-1}\right)$, and incubated at $65^{\circ} \mathrm{C}$ for $30 \mathrm{~min}$. Subsequently, $2 \mathrm{ml}$ acetone and $100 \mu \mathrm{l}$ of a $30 \%$ hydrogen peroxide solution were added and mixed well. The mixture was collected, filled up to $10 \mathrm{ml}$ with ethanol, and incubated in an ultrasonic bath at $4{ }^{\circ} \mathrm{C}$ for $45 \mathrm{~min}$. Pellets were harvested and centrifuged at $8000 \mathrm{~g}$ for $10 \mathrm{~min}$. The resulting supernatants were collected and examined for $\mathrm{CoQ}_{10}$ production.

\section{HPLC analysis}

$\mathrm{CoQ}_{10}$ production and 4HBA consumption were determined using an UltiMate TM 3000 HPLC system (Thermo-Fisher Scientific, Waltham, US) equipped with a $250 \mathrm{~mm} \times 4.6 \mathrm{~mm}$ $\times 5 \mu \mathrm{m}$, C18-reversed phase column (Thermo-Fisher Scientific, Beijing, China) and a photo-diode array detector. Methanol/ isopropyl alcohol (HPLC grade, Sigma-Aldrich, Shanghai, China) at a ratio of $3: 1(\mathrm{v} / \mathrm{v})$ was used as the mobile phase at a flow rate of $0.8 \mathrm{ml} \mathrm{min}{ }^{-1}$ and the column was kept at $30{ }^{\circ} \mathrm{C}$. $\mathrm{CoQ}_{10}$ was detected at $275 \mathrm{~nm}$ and $254 \mathrm{~nm}$. CoQ ${ }_{10}$ concentrations were determined using a standard curve based on an HPLC-grade authentic $\mathrm{CoQ}_{10}$ standard (Sigma-Aldrich, Shanghai, China). 4HBA was resolved using 15\% acetonitrile with $0.1 \%$ trifluoroacetic acid (TFA) in water at a flow rate of $0.8 \mathrm{ml} \mathrm{m^{-1 }}$, on a HyperREZ XP $300 \mathrm{~mm} \times 7.7 \mathrm{~mm} \times 8 \mu \mathrm{m}$ column (Thermo-Fisher Scientific, Beijing, China) which was kept at $65{ }^{\circ} \mathrm{C}$, and detected at $254 \mathrm{~nm}$. The concentration of 4HBA was quantified using a standard curve based on authentic standard material (Sigma-Aldrich, Shanghai, China).

\section{Results and discussion}

\section{Expression of three different PcaK homologs in $R$. sphaeroides GY-2}

The PcaK proteins derived from Acinetobacter calcoaceticus and Klebsiella pneumoniae have been functionally identified as plasma membrane transporter of 4 HBA. $^{19,26}$ Since PcaKmediated transport is active and energized by the proton motive force, we hypothesized that this protein would significantly increase 4HBA transport in other bacteria as well. In this study, three PcaK homologs, derived from Acinetobacter calcoaceticus, Klebsiella pneumoniae, and Corynebacterium glutamicum, were used to improve $\mathrm{CoQ}_{10}$ production and introduced on plasmids into $R$. sphaeroides GY-2 via the bi-parental conjugation method. However, high-level expression of PcaK led to excessive accumulation of $4 \mathrm{HBA}$, resulting in inhibition of cell growth and $\mathrm{CoQ}_{10}$ production (Fig. 1). Because tac acts as a strong promoter, the transcriptional level of the PcaK genes could be weakened when they were inserted behind an RFP open reading frame. Expression of each PcaK homolog in R. sphaeroides GY-2 was verified by PCR and western blot assays. The recombinant three $R$. sphaeroides strains expressing CgPcak, KpPcak, and AcPcak were named as RS-CgPcaK, RS-KpPcaK, and RS-AcPcaK, respectively. The $R$. sphaeroides GY-2 containing pBBR1MCS2-tac-RFP (RS-GY2) was used as the control.

As shown in Fig. 2A, the results clearly show the presence of the recombinant PcaK in each of the three $R$. sphaeroides GY-2 strains. Fig. 2B illustrates the three bands corresponding to the respective PcaK proteins, each with a molecular weight of about $32 \mathrm{kDa}$. Even though the observed molecular weight was substantially lower than the theoretical prediction of $47 \mathrm{kDa}$, it was consistent with a report by Pernstich et $a .^{26}$ The results indicate that none of the heterologous proteins were significantly modified by $R$. sphaeroides GY-2. In other words, their normal biological function should not be affected in this host organism.

\section{Effect of 4HBA on biomass accumulation}

Most Gram-positive and some Gram-negative bacteria were found to be considerably sensitive to $4 \mathrm{HBA} .{ }^{20}$ Generally, it has been observed that the growth of bacteria is markedly inhibited when the concentration of 4HBA rises above $1 \mathrm{~mol} \mathrm{l}^{-1}$. To elucidate the effect of 4HBA on the growth of $R$. sphaeroides, the cells were cultured in the specific medium supplemented with $0.5,1,2$, and $4 \mathrm{~mol} \mathrm{l}^{-1}$ of $4 \mathrm{HBA}$, respectively (Fig. 3). It was found that all three recombinant strains expressing foreign PcaK proteins were more sensitive to 4HBA than RS-GY2, which was used as control. Among the three recombinant strains, the growth of RS-CgPcaK was most susceptible to inhibition by 4HBA. The biomass yield of RS-CgPcaK was lower by $12.8-19.1 \%$ at the different 4 HBA concentrations, compared to the control. Interestingly, a low concentration of 4HBA (0.5-1 mol $\left.\mathrm{l}^{-1}\right)$ contributed to increased biomass accumulation of all the $R$. sphaeroides strains. However, biomass ceased to increase and began to decline when more than $1 \mathrm{~mol} \mathrm{l}^{-1}$ of $4 \mathrm{HBA}$ was added to the medium.

In fact, the biological function of CgPcaK, AcPcaK, and KpPcaK could be demonstrated via the increased 4HBA transport efficiencies and consumption rates exhibited by the three recombinant $R$. sphaeroides GY-2 strains. Theoretically, the PcaK with the highest transport efficiency should most effectively facilitate the transmembrane transportation of 4HBA, and consequently, the cell growth of the corresponding $R$. sphaeroides GY-2 strain should be inhibited the most. As expected, all the recombinant strains expressing PcaK showed higher rates of growth inhibition at different concentrations of $4 \mathrm{HBA}$ than the control strain (Fig. 3). Thus, it can be concluded that the PcaK proteins from different sources indeed mediate 4HBA transport in $R$. sphaeroides GY-2. CgPcaK appeared to have the highest 
(A)

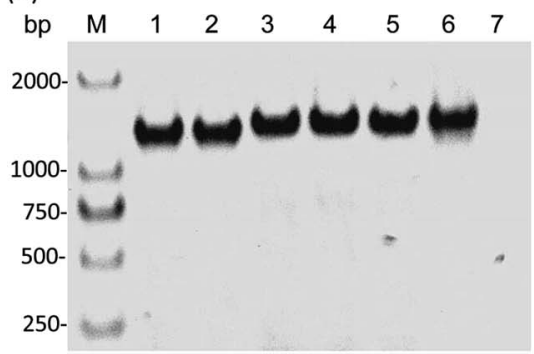

(B)

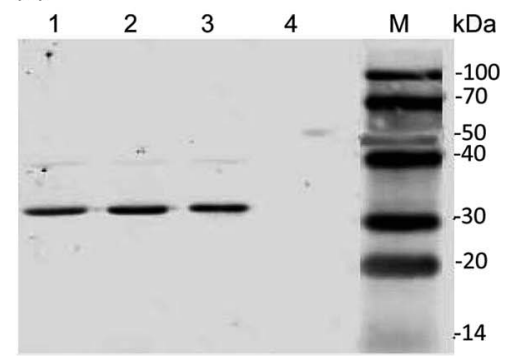

Fig. 2 Integration of three PcaK transporters into $R$. sphaeroides GY-2. (A) PCR verification of PcaK genes from the three recombinant $R$. sphaeroides GY-2 strains. Lanes 1-2 represent gene of Cg-Pcak; lanes 3-4 represent gene of Ac-Pcak; lanes 5-6 represent gene of Kp-Pcak; lane 7 represents the control; M: DNA marker. (B) Detection of PcaK in the three recombinant $R$. sphaeroides GY-2 strains by western blot. Lanes 1-3 represent CgPcaK, AcPcaK, and KpPcaK, respectively.

transport efficiency, since its growth was inhibited the most at the different initial concentrations of 4HBA. This conclusion was confirmed by the observation that RS-CgPcaK also consumed significantly more 4HBA than the control strain (Fig. 4). All the data in this study was compared using $t$-test statistics.

\section{HBA consumption by the recombinant $R$. sphaeroides}

To determine the transport efficiency of the heterologously expressed PcaK, the 4HBA consumption and utilization by the recombinant $R$. sphaeroides strains was studied. Fig. 4 shows the 4HBA consumption of the recombinant $R$. sphaeroides GY-2 strains at four different 4 HBA concentrations. The consumption was calculated based on the difference between the initial and remaining concentrations of $4 \mathrm{HBA}$ after a fixed period of time. The four $R$. sphaeroides strains exhibited a similar pattern whereby the 4HBA consumption dramatically increased at the lower concentrations of $0.5-2 \mathrm{~mol} \mathrm{l}^{-1}$, and increased slowly at 2$4 \mathrm{~mol} \mathrm{l}^{-1}$ (Fig. 4). The reduced efficiency of 4HBA utilization at

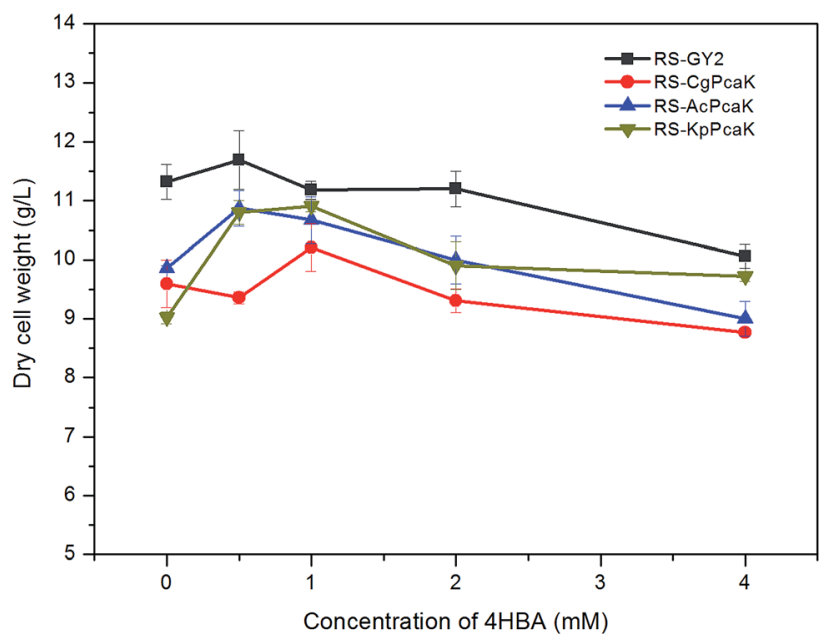

Fig. 3 Effect of different concentrations of $4 \mathrm{HBA}$ on cell growth of the recombinant $R$. sphaeroides GY-2 strains. The $R$. sphaeroides GY-2 containing pBBR1MCS2-tac-RFP (RS-GY2) was used as the control strain. Data were presented as means \pm standard deviation (SD). higher concentrations was probably due to inhibition of cellular metabolism. Both RS-CgPcaK and RS-KpPcaK consumed significantly more $4 \mathrm{HBA}$ than the control strain $(P<0.05)$ using $t$-test statistics. About $20 \%$ more $4 \mathrm{HBA}$ was transported into the cells contributing to $\mathrm{CoQ}_{10}$ biosynthesis at initial 4HBA concentrations of 1-4 $\mathrm{mol} \mathrm{l}^{-1}$. However, there was no significant difference between RS-AcPcaK and the control strain for 4HBA consumption. Interestingly, a small amount of $4 \mathrm{HBA}$ could be detected even in the culture supernatants to which no 4HBA was supplemented. The improved 4HBA consumption of RS-CgPcaK and RS-KpPcaK can be easily understood in terms of the higher 4HBA trans-membrane transport efficiency of CgPcaK and KpPcaK compared to AcPcaK.

\section{$\mathrm{CoQ}_{10}$ production}

Batch fermentations were carried out in shake flasks to determine the specific contents of $\mathrm{CoQ}_{10}$ produced by the recombinant $R$. sphaeroides strains. The results illustrate that enhanced $\mathrm{CoQ}_{10}$ productivity has indeed been achieved in the RS-CgPcaK and RS-KpPcaK strains at an initial 4HBA concentration of $0.5 \mathrm{~mol} \mathrm{l}^{-1}$, and was significantly higher than what was

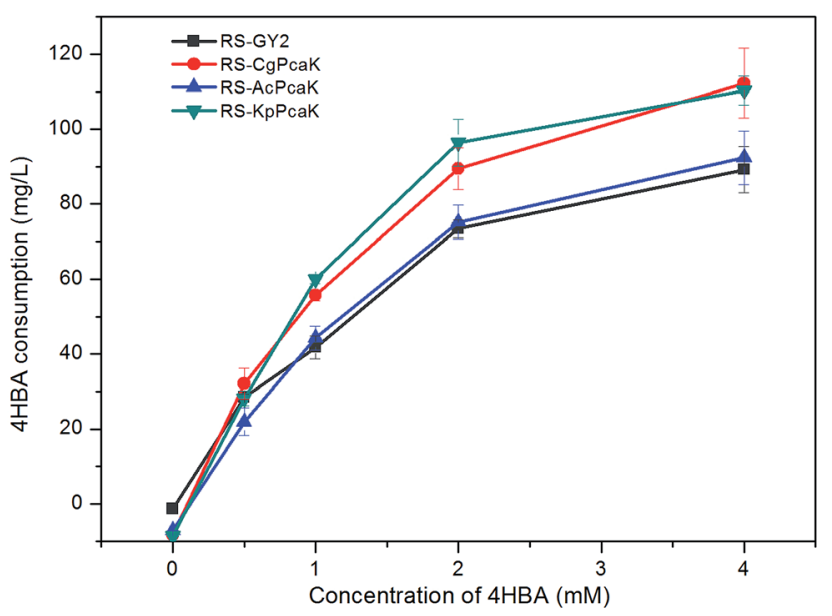

Fig. 4 4HBA consumption of the recombinant $R$. sphaeroides GY-2 strains. RS-GY2 was used as the control strain. Data were presented as means \pm standard deviation (SD). 
observed for any other concentration $(P<0.05)$ (Fig. 5B and D). After anaerobic fermentation for $48 \mathrm{~h}$, the highest $\operatorname{CoQ}_{10}$ productivity values for RS-CgPcaK and RS-KpPcaK reached $17.45 \mathrm{mg} \mathrm{g}^{-1}$ DCW and $18.06 \mathrm{mg} \mathrm{g}^{-1} \mathrm{DCW}$, respectively. In contrast, 4HBA supplementation did not contribute to improved $\mathrm{CoQ}_{10}$ productivity in either the RS-AcPcaK strain or the RS-GY2 control strain (Fig. 5A and C). Furthermore, the $\mathrm{CoQ}_{10}$ productivity of the four $R$. sphaeroides GY-2 strains decreased rapidly at higher levels $\left(\geq 1 \mathrm{~mol} \mathrm{l}^{-1}\right)$ of $4 \mathrm{HBA}$, probably due to suppressed cell growth. Taken together, the results indicate that above a certain threshold, the higher the initial concentration of $4 \mathrm{HBA}$ that was added, the lower the $\mathrm{CoQ}_{10}$ content that was obtained.

The results in Fig. 5 illustrate the increased 4HBA consumption led to enhanced $\mathrm{CoQ}_{10}$ productivity. This is consistent with a previously published report that $\mathrm{CoQ}_{10}$ production could be improved by increasing the flux of precursors to the ubiquinone pathway in an engineered $E$. coli strain. ${ }^{2}$ RS-AcPcaK likely transports the most 4HBA into the cytoplasm, judging by the most significant inhibition of biomass accumulation compared to other recombinant $R$. sphaeroides strains (Fig. 3). However, we unexpectedly found that RS-AcPcaK failed to increase $\mathrm{CoQ}_{10}$ biosynthesis and $4 \mathrm{HBA}$ consumption. It is possible that the highly concentrated 4HBA transported by RS-AcPcaK is transformed to the inhibitory dihydroxybenzoic acid by undergoing ortho ring cleavage via the $\beta$-ketoadipate pathway, ${ }^{\mathbf{1 8}}$ suggesting that the precursor flux to the ubiquinone pathway was not significantly increased and $\mathrm{CoQ}_{10}$ production was therefore also not enhanced.

High intracellular concentrations of aromatic carboxylic acids are not persistent in bacteria, since there is a highlyregulated efflux system that maintains the intracellular balance of $4 \mathrm{HBA}$ in $E$. coli and other microorganisms. ${ }^{27}$ Accordingly, when high-levels of $4 \mathrm{HBA}$ were transported into the $R$. sphaeroides cells, expression of the efflux system was likely activated, resulting in the removal of excess 4HBA and restoration of cellular homeostasis. In this study, a small amount (less than $8 \mathrm{mg} \mathrm{l}^{-1}$ ) of 4HBA was found in the culture supernatants not supplemented with external 4HBA, indicating that not all of the endogenously synthesized 4HBA was channeled into the ubiquinone pathway for $\mathrm{CoQ}_{10}$ production. As an intermediate, the transported 4HBA which accumulated to high intracellular levels prompted the reactions in the ubiquinone pathway. However, it was observed in this study that only an initial concentration of less than $0.5 \mathrm{mM} 4 \mathrm{HBA}$ enhanced the biosynthesis of $\mathrm{CoQ}_{10}$ in $R$. sphaeroides. Excessive amounts of intracellular 4HBA are not only directly detrimental to cell growth and biomass accumulation, but probably need to be pumped out of the cells by the efflux system, introducing an additional energy drain.
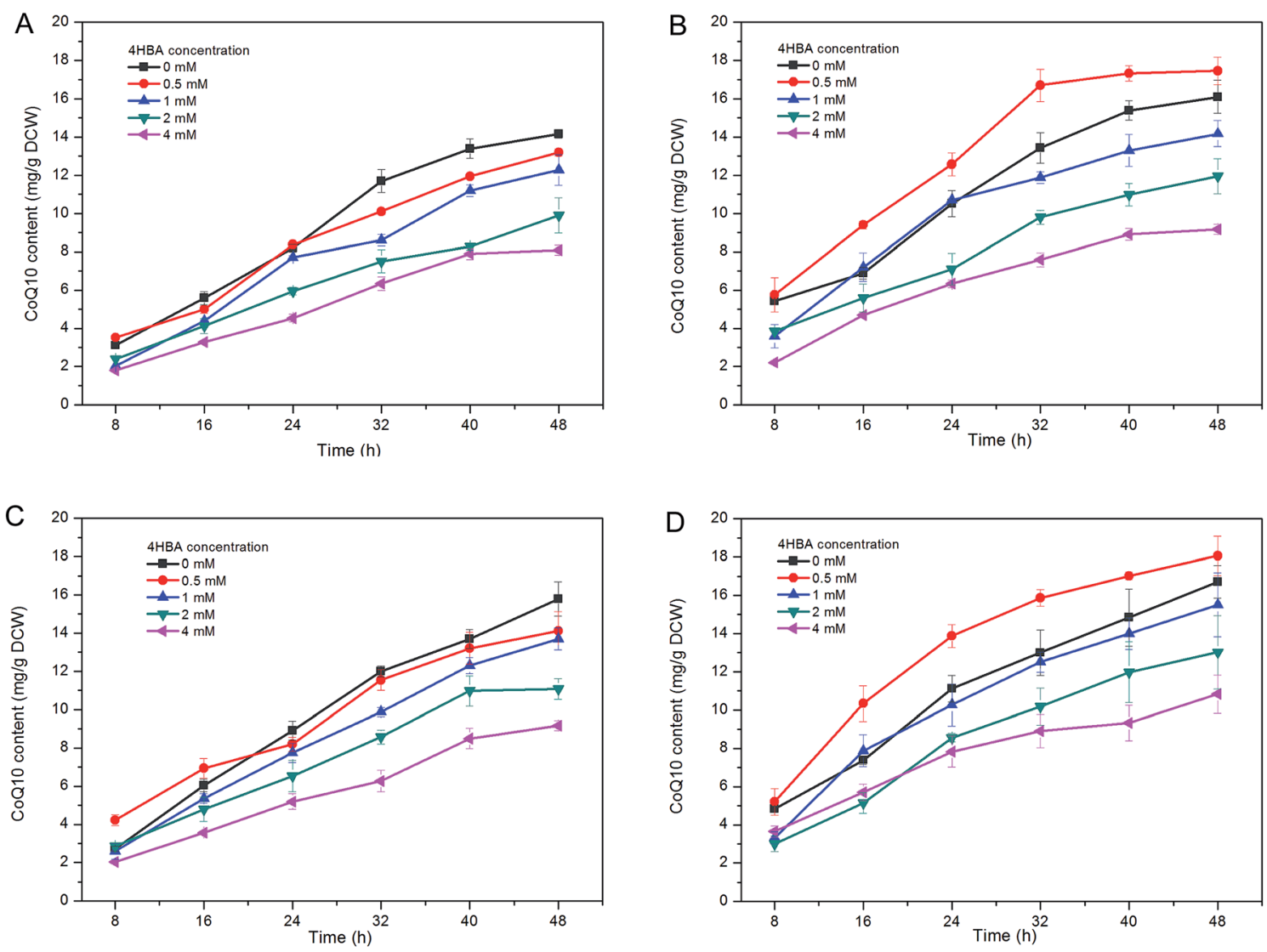

Fig. 5 Batch fermentation results of the specific contents of $\mathrm{CoQ}_{10}$ produced by the four recombinant $R$. sphaeroides $G Y-2$ strains at different concentration of 4HBA. (A) RS-GY2 (B) RS-CgPcaK (C) RS-AcPcaK (D) RS-KpPcaK. Data were presented as means \pm standard deviation (SD). 


\section{Conclusions}

Three membrane transport proteins, AcPcaK, КрPcaK, and CgPcaK, were individually heterologously expressed in $R$. sphaeroides GY-2 to enhance the uptake of extracellular 4HBA, which is an important intermediate for $\mathrm{CoQ}_{10}$ synthesis. The transport efficiency and consumption of $4 \mathrm{HBA}$ by these three recombinant $R$. sphaeroides GY-2 strains has been studied. To our best knowledge, this is the first reported instance of improved supplementation of an intermediate of the ubiquinone pathway to enhance $\mathrm{CoQ}_{10}$ production in the natural producer $R$. sphaeroides. This study confirms the effectiveness of the strategy used for improving $\mathrm{CoQ}_{10}$ production and will broaden its applications for further improvements.

\section{Acknowledgements}

This work was financially supported by National Natural Science Foundation of China (No. 21406130) and Natural Science Foundation of Fujian Province of China (No. 2016J01148; 2016J01147).

\section{Notes and references}

1 W. M. Bader, T. Xie, C. A. Yu and J. C. Bardwell, J. Biol. Chem., 2000, 275, 26082-26088.

2 C. P. Cluis, A. Ekins, L. Narcross, H. Jiang, N. D. Gold, A. M. Burja and V. J. Martin, Metab. Eng., 2011, 13, 733-744. 3 F. L. Crane, J. Am. Coll. Nutr., 2001, 20, 591-598.

4 D. A. Groneberg, B. Kindermann, M. Althammer, M. Klapper, J. Vormann, G. P. Littarru and F. Doring, Int. J. Biochem. Cell Biol., 2005, 37, 1208-1218.

5 H. S. Zahiri, S. H. Yoon, J. D. Keasling, S. H. Lee, K. S. Won, S. C. Yoon and Y. C. Shin, Metab. Eng., 2006, 8, 406-416.

6 J. M. Hodgson, G. F. Watts, D. A. Playford, V. Burke and K. D. Croft, Eur. J. Clin. Nutr., 2002, 56, 1137-1142.

7 H. N. Bhagavan and R. K. Chopra, Clin. Nutr., 2005, 24, 331-338. 8 C. Schmelzer, I. Lindner, C. Vock, K. Fujii and F. Doring, IUBMB Life, 2007, 59, 628-633.

9 G. S. Choi, Y. S. Kim, J. H. Seo and Y. W. Ryu, Process Biochem., 2005, 40, 3225-3229.
10 N. B. Kien, I. S. Kong, M. G. Lee and J. K. Kim, J. Ind. Microbiol. Biotechnol., 2010, 37, 521-529.

11 W. Lu, L. Ye, H. Xu, W. Xie, J. Gu and H. Yu, Biotechnol. Bioeng., 2014, 111, 761-769.

12 W. Lu, Y. Shi, S. He, Y. Fei, K. Yu and H. Yu, Biochem. Eng. J., 2013, 72, 42-47.

13 S. J. Kim, M. D. Kim, J. H. Choi, S. Y. Kim, Y. W. Ryu and J. H. Seo, Appl. Microbiol. Biotechnol., 2006, 72, 982-985.

14 Y. C. Park, S. J. Kim, J. H. Choi, W. H. Lee, K. M. Park, M. Kawamukai, Y. W. Ryu and J. H. Seo, Appl. Microbiol. Biotechnol., 2005, 67, 192-196.

15 X. Lv, H. Xu and H. Yu, Appl. Microbiol. Biotechnol., 2012, 97, 2357-2365.

16 M. J. Seo, M. C. Kook and S. O. Kim, J. Microbiol. Biotechnol., 2012, 22, 230-233.

17 Y. Yuan, Y. Tian and T. Yue, J. Biomed. Biotechnol., 2012, 2012, 607329.

18 N. N. Nichols and C. S. Harwood, J. Bacteriol., 1997, 179, 5056-5061.

19 J. L. Allende, M. Suarez, M. Gallego and A. Garrido-Pertierra, Arch. Biochem. Biophys., 1993, 300, 142-147.

20 J. Y. Cho, J. H. Moon, K. Y. Seong and K. H. Park, Biosci., Biotechnol., Biochem., 1998, 62, 2273-2276.

21 M. W. Wood, M. A. Jones, P. R. Watson, S. Hedges, T. S. Wallis and E. E. Galyov, Mol. Microbiol., 1998, 29, 883-891.

22 M. E. Kovach, P. H. Elzer, D. S. Hill, G. T. Robertson, M. A. Farris, R. M. II Roop and K. M. Peterson, Gene, 1995, 166, 175-176.

23 S. L. Porter, G. H. Wadhams and J. P. Armitage, Methods Enzymol., 2007, 423, 392-413.

24 M. D. Moore and S. Kaplan, J. Bacteriol., 1992, 174, 15051514.

25 D. A. D'Argenio, A. Segura, W. M. Coco, P. V. Bünz and L. N. Ornston, J. Bacteriol., 1999, 181, 3505-3515.

26 C. Pernstich, L. Senior, K. A. MacInnes, M. Forsaith and P. Curnow, Protein Expression Purif., 2014, 101, 68-75.

27 T. K. Van Dyk, L. J. Templeton, K. A. Cantera, P. L. Sharpe and F. S. Sariaslani, J. Bacteriol., 2004, 186, 7196-7204. 